\title{
An Instantiability Index for Intra-operative Tracking of 3D Anatomy and Interventional Devices
}

\author{
Su-Lin Lee ${ }^{1}$, Celia Riga ${ }^{2,3}$, Lisa Crowie ${ }^{2}$, Mohamad Hamady ${ }^{2}$, \\ Nick Cheshire ${ }^{2,3}$, and Guang-Zhong Yang ${ }^{1}$ \\ ${ }^{1}$ The Hamlyn Centre for Robotic Surgery \\ ${ }^{2}$ Regional Vascular Unit, St Mary's Hospital \\ ${ }^{3}$ Academic Division of Surgery, \\ Imperial College London, UK \\ \{su-lin.lee,g.z.yang\}@imperial.ac.uk
}

\begin{abstract}
The tracking of tissue deformation, imaging probes and surgical instruments is an integral part of intra-operative surgical guidance. While the recent introduction of shape instantiation provides a systematic framework for tracking 3D anatomy in real-time, deviations to the desired imaging location can affect the accuracy of the predicted shape. To examine the sensitivity of the prescribed image planes to such errors, we introduce in this paper a new Instantiability Index for providing the intrinsic level of robustness while using such scan planes for the tracking of anatomy and interventional devices. Optimisation of the Index is applied to $3 \mathrm{D}$ anatomical reconstruction and the localisation of an intraoperative imaging device. Results are shown on detailed phantom experiments for both real-time 3D shape instantiation and imaging catheter tracking.
\end{abstract}

Keywords: instantiability index, shape instantiation, tracking, localization.

\section{Introduction}

For intraoperative surgical guidance, effective tracking of tissue deformation and interventional devices is an integral part of the workflow. Despite increasing advances of intra-operative imaging techniques, particularly intra-operative MR and CT, the acquisition of real-time 3D sequences is often limited by practical constraints such as temporal resolution and potential radiation burden. For anatomical structures undergoing large tissue deformation, instantiating the entire dynamic 3D shape from limited imaging planes or sparsely sampled data becomes a pertinent research topic. The recent introduction of shape instantiation reduces the amount of real-time imaging data required to reconstruct an entire 3D shape by using models built preoperatively. For example, the work of Lee et al. [1] proposed the use of optimal scan planning for the instantiation of the organ of interest using partial least squares regression during surgery. The work balances the information content of an individual or a set of image planes against practical constraints such as available acquisition windows for capturing rapid shape deformation or transit of a contrast bolus. For intra-vascular intervention, this approach is advantageous due to a reduction in both 
the use of contrast agents to minimise nephrotoxicity and exposure to radiation. In the existing work reported thus far, the issue of errors in localisation of the scan plane, however, has not been explicitly addressed. Despite the availability of the optimal scan planes, in practice, it is not always possible to accurately localise to these plans due to co-registration errors or patient motion. This difficulty is particularly evident with handheld imaging probes such as ultrasound. With the original framework of shape instantiation, it is assumed that the captured scan plane will conform to the optimal one defined. Deviation to the prescribed optimal scan plane(s) or errors induced due to anatomical access difficulties can lead to significant errors in shape instantiation. It is therefore desirable to consider not only the information content of a given image plane in terms of its shape instantiability, but also the underlying resilience to perturbations or localisation errors. This situation is illustrated schematically in Fig. 1 where the robustness of two different scan planes is shown via their shape instantiation results.
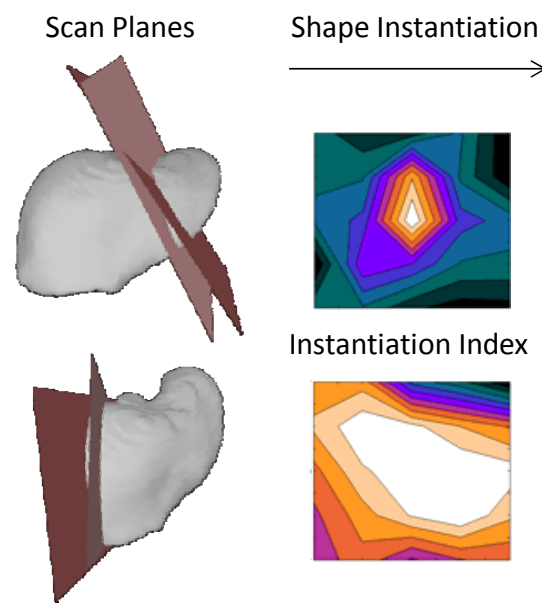

Instantiation Index
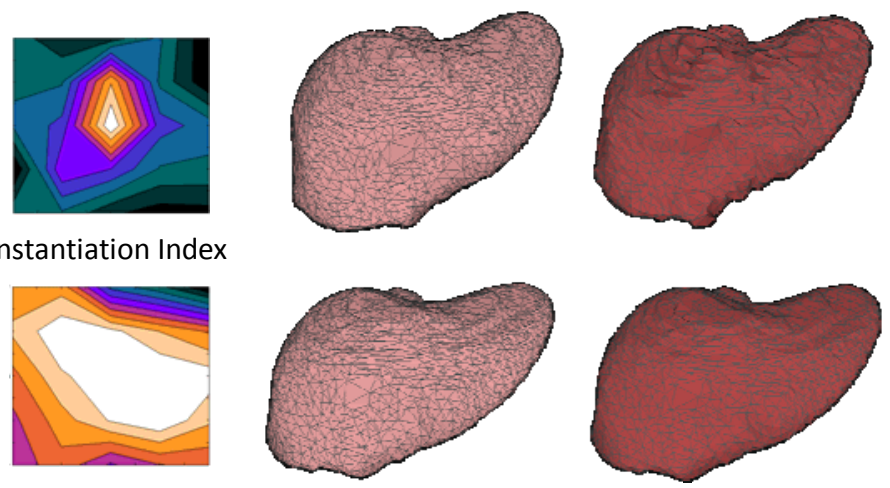

Fig. 1. The use of the Instantiability Index with respect to the existing shape instantiation framework, here with a liver example. An examination of the instantiation index for a scan plane indicates how a perturbation in its position affects the instantiation: (top) a scan plane that is less and (bottom) one that is more robust.

The purpose of this paper is to introduce a new shape Instantiability Index for accurate and more robust intra-operative 3D shape instantiation. We will demonstrate its practical use for both $3 \mathrm{D}$ anatomical reconstruction and reliable tracking of interventional devices. For the former, we will illustrate the underlying reasons of introducing the shape instantiability index and how it reflects the potential errors in shape instantiation caused by deviations/perturbations from the defined scan planes whereas for the latter, we demonstrate its novel use for the tracking of IVUS catheters during intravascular interventions. The tracking of IVUS catheters is known to be difficult without the use of additional positional sensors or repeated contrast-enhanced $\mathrm{x}$-ray guidance and methods based on unscented particle filters [2] and image based 
co-registration [3] have been proposed. The method introduced in this paper represents an alternative approach that allows simultaneous geometrical recovery and device tracking.

For detailed quantitative evaluation, the use of the Index to optimise the position of the scan plane for shape instantiation was demonstrated with a deformable silicone liver phantom. The scan plane was optimised, used to reinitialise the framework, and used to instantiate the liver model. The Index was also used to solve the inverse problem of finding the position of an IVUS catheter in situ, as demonstrated with a silicone arterial model.

\section{Methods}

\subsection{Instantiability Index for Shape Recovery}

The basic concept of shape instantiation can be described as follows. Prior to an operation, a statistical shape model can be built from preoperative images and an optimal scan plane determined, along which the most information of the entire shape is derived. The model is trained (e.g., through the use of partial least squares regression) with this data and further image planes obtained intraoperatively are input to the regression which predicts the entire organ shape in real-time.

The Instantiability Index is based on the comparison of the real-time image data to the contours of the preoperative model along the scan plane. An examination of the Index for the scan plane during perturbation gives an indication of the sensitivity of the plane, i.e., how prone to errors a small perturbation to the plane parameters can cause. A stable Index indicates a robust scan plane, with a small error in the plane orientation or position not affecting the shape instantiation. A sharp peak in the Index typically indicates a unique solution but the corresponding scan plane will be sensitive to error. Albeit undesirable for shape instantiation, this location can be used to localise the position of an in vivo imaging probe, as it suggests the local geometry is unique.

With the proposed framework, contours $P$ are found by the intersection of the scan plane with the preoperative shape model, where the plane $p$ is defined with an origin $o$ and a normal $n$. Geometrically, the preoperative surface mesh consists of triangles defining the geometry of the organ of interest. The contours are then projected to $2 \mathrm{D}$ for comparison to the real-time imaging data. With two $3 \mathrm{D}$ vectors $x$ and $y$, from the image origin $o$, along the respective axes of the 2D image, the 2D coordinates of any $3 \mathrm{D}$ point $a$ are:

$$
(u, v)=\{(a-o) \cdot x,(a-o) \cdot y\}
$$

Preprocessing of the ultrasound images was performed prior to optimisation. The objective function to be minimised, defined as the Instantiability Index (II), is then

$$
I I=f(R, T)=-\sum_{i=0}^{N}\left(I_{i} \times\left(R P_{i}+T\right)\right)
$$


where $I_{i}$ is the intensity of the image at position $i, R$ and $T$ are the rotation and translation of the scan plane (the parameters to be optimised), and $P$ is the intersection between the scan plane and the preoperative model (with $\mathrm{N}$ points in the contour). A comparison of optimisation methods was performed with Simulated Annealing [4] performing most reliably. With the optimised plane, the partial least squares regression was reinitialised semi-automatically; the preoperative statistical shape model was recut and the points on the contour resampled. This updated partial least squares regression was used to predict the shape.

\subsection{Data Collection}

A number of calibration techniques and corresponding phantoms have been developed for freehand 3D ultrasound systems [5]. For this work, a three-point crossed-wires phantom was built for the calibration of intra-operative ultrasound images (coordinate system $U$ ) to the coordinate space of a tracking device (coordinate system $N$ ). Likewise, the transforms were also found which mapped the coordinate space of an electromagnetic tracker to that of the CT imaging space (coordinate system $C$ ), calculated using PRAXIS [6]. This then results in a vector defining translation and a quaternion for rotation between the ultrasound image points to the original CT imaging space. Using the notation from Prager et al.[7], this results in the following calculation: ${ }^{C} x={ }^{C} T_{N}{ }^{N} T_{U}{ }^{U} x$, where ${ }^{C} T_{N}$ is the transformation from the coordinate system $C$ to $N$ and ${ }^{N} T_{U}$ is that from $U$ to $N$.

For detailed validation of the proposed method, ultrasound images of a silicone organ phantom [8] were collected; this near life-sized phantom consists of a number of silicone organs, including modified lungs with inserts to simulate respiration and hence deform the silicone liver. Translation of the liver was not achieved. The data were collected on an ALOKA prosound $\alpha 10$ system (Aloka Co. Ltd, Tokyo, Japan). A 2D convex probe, with a custom built mount for a passive infrared marker to be tracked by an NDI Polaris tracker (Northern Digital, Inc, Waterloo, ON, Canada), was used. Calibration between the two coordinate systems is described in the next section. The ultrasound images were captured using a PC video capture card connected to the S-video output feed of the scanner. Scans were obtained at 7 different liver positions.

To demonstrate the use of the proposed instantiability index for tracking in vivo imaging probes, IVUS images were acquired on a Volcano IVUS system (Volcano, San Diego, CA, USA) at an anonymous hospital. An Elastrat (Elastrat Sarl, Geneva, Switzerland) pulsatile silicone phantom of the aortic arch was imaged with IVUS and with a corresponding CT roll scan with a Philips Allura Xper CT scanner. A single fluoroscopic image was also captured to provide an initial position for the IVUS catheter tip; as both scans were obtained on the same scanner, no explicit registration was required. Ten catheter positions were collected. Analyze (AnalyzeDirect, Inc, Overland Park, KS, USA) was used to perform the segmentation and model building. For the IVUS data, the position of the tip of the catheter was initialised by obtaining its rough position in two dimensions from the corresponding fluoroscopic image. The optimisation of the instantiability index was then used to determine the final position of the catheter within the mesh. 


\section{Results}

\subsection{Validation for Shape Instantiation}

For robust anatomical shape instantiation with data collected from the Aloka scanner, the scan plane was first optimised with respect to the preoperative model. With this optimised scan plane, partial least squares regression for the shape instantiation was reinitialised. The errors were calculated as the mean of the distances from the points on the instantiated shape to the closest points on the ground truth model, the 3D mesh of the liver as manually segmented from CT images.

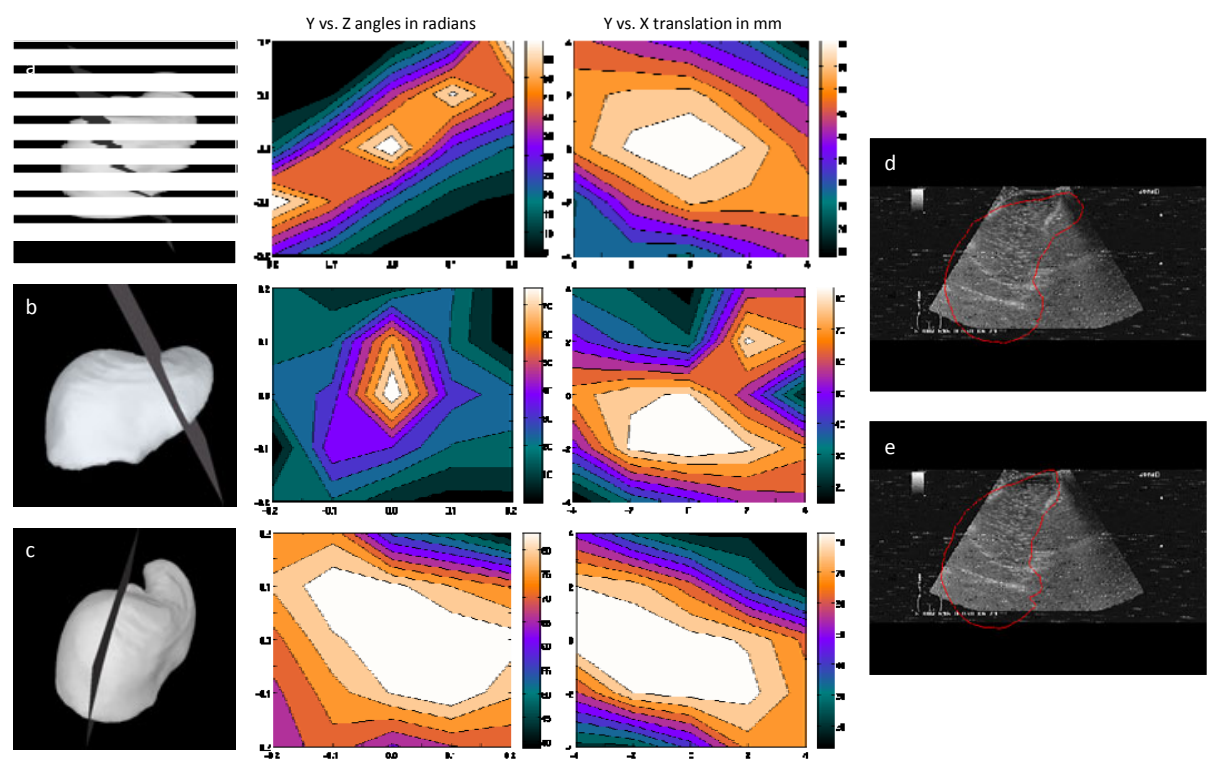

Fig. 2. (a, b, c) The Instantiation Index for the scan plane at three locations with respect to the silicone liver model. Optimisation for respiratory position 1 with the Aloka ultrasound image with (d) the initial contour and (e) the optimised contour with the first scan plane (a) as the optimised scan plane.

An examination of the Instantiability Index, with translation and rotation of the plane, for three different scan planes is shown in Fig. 2. The graphs show that a small change in translation of the plane is unlikely to affect the shape instantiation but a change in the orientation might, highlighting the need for scan plane optimisation. The third scan plane is the most forgiving to any perturbations but for this phantom, is inaccessible with the ultrasound probe. The result of the optimisation of a contour with an Aloka ultrasound image of the silicone phantom is also shown in Fig. 2. Fig. 3 demonstrates the results of the instantiation (mean error with standard deviation) when using the ultrasound data along the optimised scan plane versus the original scan plane. The errors are less when using the reinitialised shape instantiation method 


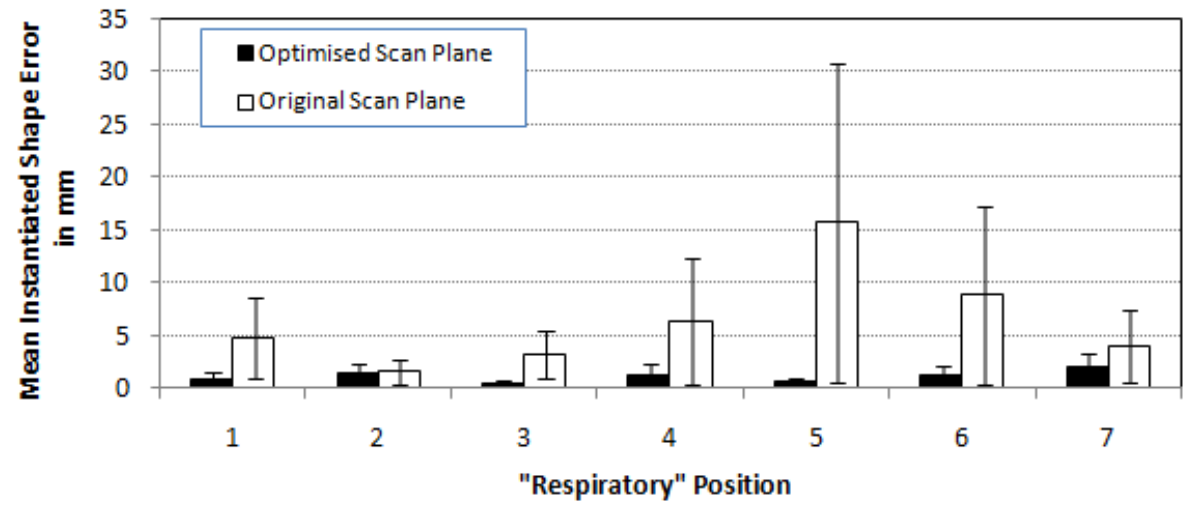

Fig. 3. The optimised instantiation mean errors, and standard deviations, and instantiation with the collected data at the original scan plane mean errors to the ground truth. The respiratory positions are numbered based on the number of lung inserts in the silicone model.

than when using the original scan plane. In the case of Position 5, the instantiation using the original scan plane failed, highlighting the necessity of optimising the scan plane intraoperatively.

\subsection{Validation for Intraoperative Catheter Localisation}

The setup for the IVUS experiment with the vascular silicone phantom can be seen in Fig. 4. For this second set of the IVUS data, the optimal location of the IVUS imaging probe was compared to that found in the ground truth CT images. A comparison of the graphs of the Instantiability Index, as the plane undergoes variation, at the descending aorta and near a bifurcation is shown in Fig. 5. A sharp peak is evident in the latter example, making that scan plane ideal for transducer localisation. Fig. 5 also shows the result of the optimisation of the model contours for Position 2 along with the final position of the imaging plane with respect to the geometry of the silicone vascular phantom. The error in the position of the IVUS catheter, calculated as the

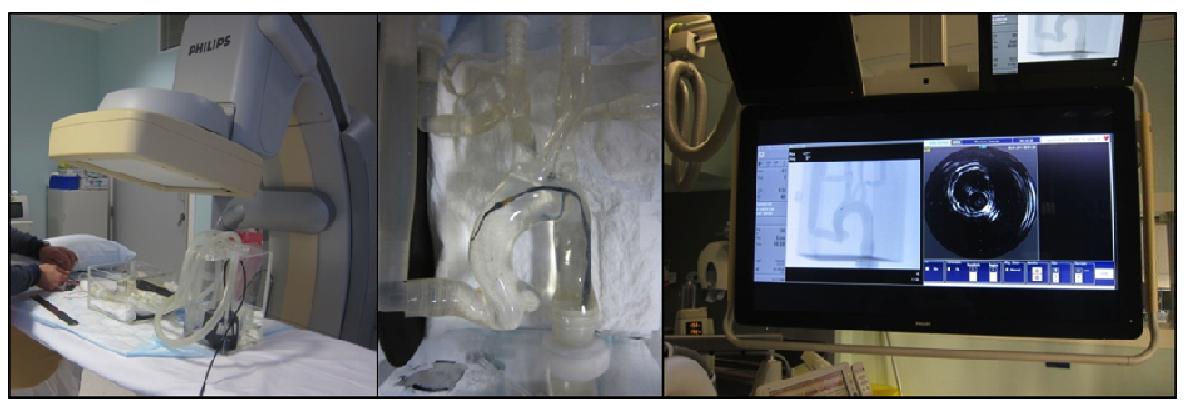

Fig. 4. The IVUS experiment setup. From left to right, the pulsatile silicone vascular phantom within the Philips scanner, the phantom with an IVUS catheter inserted, and the fluoroscopic image displayed with the IVUS image. 
Euclidean distance between the calculated position and the ground truth found in the CT scan, across all 10 positions is shown in Table 1, along with their corresponding Instantiability Index Measures, with a higher measure indicating a graph with a greater peak. The errors were found to be generally less than $4 \mathrm{~mm}$, with the exception of Position 4, when the IVUS catheter was located in one of the smaller branches of the phantom.
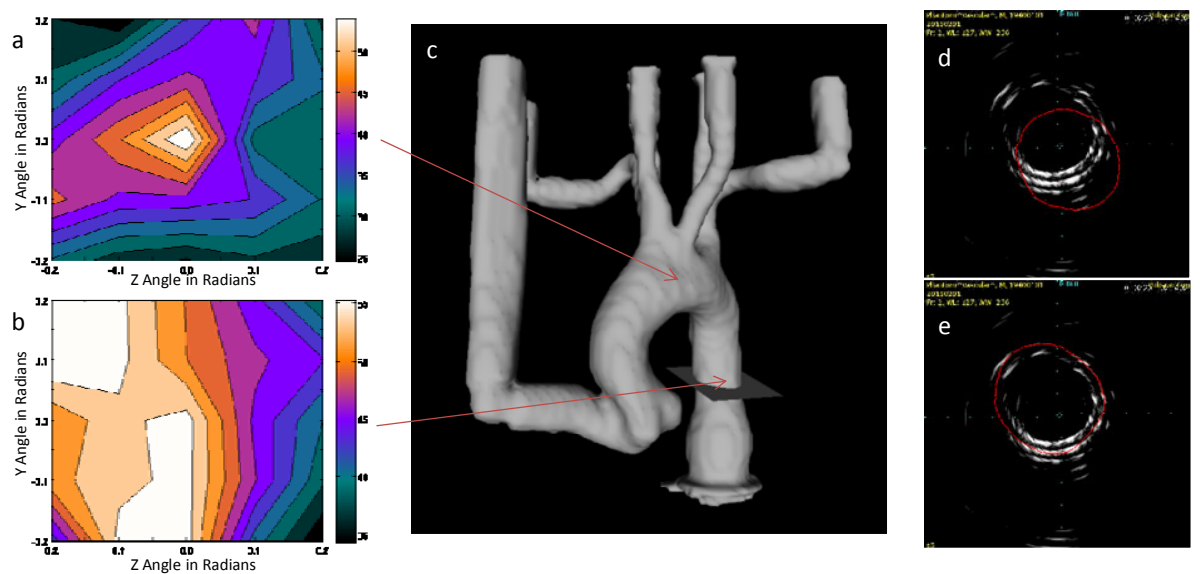

Fig. 5. The Instantiation Index (a) near a bifurcation of the artery and (b) at the descending aorta (where the lumen remains similar despite variations in the plane). Optimisation for Position 2 with the pre-processed IVUS image (d) with the initial contour overlaid, (e) the optimised contour, and (c) the optimised scan plane with the vascular phantom mesh.

Table 1. The errors in mm, along with the Instantiation Index Measure, of the estimated IVUS transducer position for the 10 different catheter positions in the silicone vascular phantom

\begin{tabular}{llllll}
\hline \hline Position & 1 & 2 & 3 & 4 & 5 \\
\hline Error & 3.05 & 2.32 & 2.58 & 6.86 & 3.20 \\
\hline IIM & 0.97 & 1.19 & 1.08 & 1.10 & 1.52 \\
\hline \hline Position & 6 & 7 & 8 & 9 & 10 \\
\hline Error & 2.01 & 1.83 & 3.70 & 4.08 & 1.14 \\
\hline IIM & 1.48 & 1.27 & 1.23 & 1.07 & 1.49 \\
\hline \hline
\end{tabular}

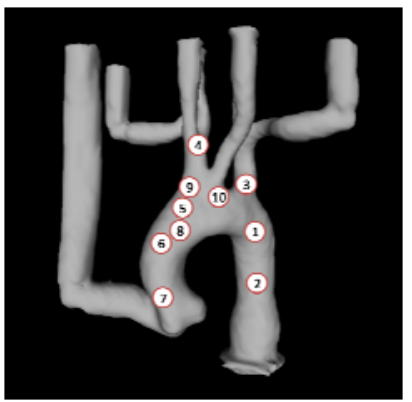

With both silicone phantoms, there were many similar areas and hence many local minima where an optimisation method could be trapped. Simulated annealing was found to be the most successful optimisation technique for both datasets due to its ability to step out of local minima. However, it does not currently run in real-time. 


\section{Discussion and Conclusion}

In this paper, we have proposed a new index for shape instantiation. We have demonstrated its use for both intraoperative anatomical reconstruction and probe tracking. The proposed instantiation index provides a measure of the amount of information in the scan plane to reduce the errors in instantiation and localisation. Local perturbations of the scan plane can be examined and a region of stable Indices is suitable for shape instantiation, with the use of an optimisation method to determine the exact plane captured with respect to the preoperative model. This optimised plane is used to reinitialise the predictive model, thus making prediction of the entire shape more accurate intraoperatively. A scan plane with a distinctive peak in the index, with respect to nearby scan planes, indicates a global optimum at that plane and hence is suitable for localisation within the preoperative model. The paper addresses a practical aspect of shape instantiation that was not considered in previous work and through the use of a novel shape instantiation index, improves both morphological reconstruction and device tracking.

Acknowledgments. The authors would like to thank Alessio Dore for his assistance in data collection.

\section{References}

1. Lee, S.-L., Chung, A., Lerotic, M., Hawkins, M.A., Tait, D., Yang, G.-Z.: Dynamic Shape Instantiation for Intra-operative Guidance. In: Jiang, T., Navab, N., Pluim, J.P.W., Viergever, M.A. (eds.) MICCAI 2010. LNCS, vol. 6361, pp. 69-76. Springer, Heidelberg (2010)

2. Brij Koolwal, A., Barbagli, F., Carlson, C., Liang, D.: An Ultrasound-based Localization Algorithm for Catheter Ablation Guidance in the Left Atrium. The International Journal of Robotics Research 29, 643-665 (2010)

3. King, A.P., Rhode, K.S., Ma, Y., Yao, C., Jansen, C., Razavi, R., et al.: Registering Preprocedure Volumetric Images With Intraprocedure 3-D Ultrasound Using an Ultrasound Imaging Model. IEEE Transactions on Medical Imaging 29, 924-937 (2010)

4. Press, W.H., Teukolsky, S.A., Vetterling, W.T., Flannery, B.P.: Numerical Recipes in C. Cambridge University Press, Cambridge (1992)

5. Mercier, L., Lang $\varnothing$, T., Lindseth, F., Collins, D.L.: A review of calibration techniques for freehand 3-D ultrasound systems. Ultrasound in Medicine \& Biology 31, 449-471 (2005)

6. Gegenfurtner, K.: PRAXIS: Brent's algorithm for function minimization. Behavior Research Methods 24, 560-564 (1992)

7. Prager, R.W., Rohling, R.N., Gee, A.H., Berman, L.: Rapid Calibration for 3-D Freehand Ultrasound. Ultrasound in Medicine and Biology 24, 855-869 (1998)

8. Lerotic, M., Lee, S.-L.: A Multimodal Silicone Phantom for Robotic Surgical Training and Simulation. In: The Hamlyn Symposium on Medical Robotics, London, UK, pp. 65-66 (2010) 